\title{
COMMUTING UNILATERAL SHIFTS
}

\author{
JAMES A. DEDDENS ${ }^{1}$
}

Abstract. Examples are given to show that two commuting unilateral shifts (of dimension $\infty, \infty$ or $m, n$ with $2 \leqq m, n<\infty$ ) need not commute with a unilateral shift of multiplicity one.

An operator $V$ on a Hilbert space $\mathcal{H C}$ is called a unilateral shift of multiplicity $k$ if $V$ is an isometry such that $\bigcap_{n=1}^{\infty} V^{n} \mathfrak{F C}=\{0\}$ and $\operatorname{dim}(\mathcal{H C} \ominus V \mathcal{H})=k$ (see [4], [5]). Every unilateral shift of multiplicity one is unitarily equivalent $(\cong)$ to $M_{e}$ on $H^{2}$ (where $\left(M_{e} f\right)\left(e^{i \theta}\right)=e^{i \theta} f\left(e^{i \theta}\right)$ for $f \in H^{2}$ (circle) ). $\phi \in H^{2}$ is called an inner function if $\left|\phi\left(e^{i \theta}\right)\right|=1$ a.e.

Proposition (See Corollary 3 in [1]). A family of commuting unilateral shifts $\left\{V_{\alpha}\right\}_{\alpha \in A}$ can be represented as multiplications by inner functions on $\mathrm{H}^{2}$ if and only if there is a unilateral shift of multiplicity one that commutes with all the $V_{\alpha}$.

In studying the structure of families of commuting isometries, one would like to know whether the preceding proposition describes all families of commuting unilateral shifts. We show that this is not the case by exhibiting commuting unilateral shifts that do not commute with a unilateral shift of multiplicity one. We would like to thank Professor R. G. Douglas for suggesting the above problem. Of course, every unilateral shift commutes with a unilateral shift of multiplicity one.

Let $\boldsymbol{Z}^{+2}$ be the product of two copies of the nonnegative integers. For any Hilbert space $\mathscr{K}$, we define $l^{2}\left(\boldsymbol{Z}^{+2}, \mathfrak{K}\right)$ to be the Hilbert space of square summable functions from $\boldsymbol{Z}^{+2}$ into $\mathcal{K}$. Consider operators $V_{1}$ and $V_{2}$ on $l^{2}\left(\boldsymbol{Z}^{+2}, \mathcal{K}\right)$ defined by

$$
\begin{aligned}
\left(V_{1} a\right)_{n, m} & =a_{n-1, m} & & \text { if } n \geqq 1, \\
& =0 & & \text { if } n=0
\end{aligned}
$$

and

Received by the editors September 25, 1969.

AMS Subject Classifications. Primary 4710, 4725, 4750.

Key Words and Phrases. Inner functions, Blaschke products, backward translation, isometry.

1 This paper is part of the author's doctoral thesis written under the direction of Dr. Peter A. Fillmore at Indiana University. 


$$
\begin{aligned}
\left(V_{2} a\right)_{n, m} & =a_{n, m-1} & & \text { if } m \geqq 1, \\
& =0 & & \text { if } m=0,
\end{aligned}
$$

for $a=\left(a_{n, m}\right) \in l^{2}\left(\boldsymbol{Z}^{+2}, \Re\right)$. Then $V_{1}$ and $V_{2}$ are commuting unilateral shifts of infinite multiplicity such that $V_{1}$ commutes with $V_{2}^{*}$. Hence by Theorem 9 in [1] $V_{1}$ and $V_{2}$ cannot commute with a unilateral shift of multiplicity one. We remark that any two commuting unilateral shifts $U_{1}, U_{2}$ that satisfy $U_{1}$ commutes with $U_{2}^{*}$ are unitarily equivalent to the above operators for some Hilbert space $\Re$ [2].

For any two integers $2 \leqq n, m<\infty$, we now construct commuting unilateral shifts of multiplicity $n$ and $m$, which do not commute with a unilateral shift of multiplicity one. We assume $2 \leqq n \leqq m<\infty$. Define $A_{0}$ and $A_{1}$ on $\mathcal{K} \equiv n$-dimensional complex space by

$$
A_{0}\left(y_{1}, y_{2}, \cdots, y_{n}\right)=\left(0, y_{1}, \cdots, y_{n-1}\right)
$$

and

$$
A_{1}\left(y_{1}, y_{2}, \cdots, y_{n}\right)=\left(y_{n}, 0,0, \cdots, 0\right) .
$$

We then define $V_{n}$ and $V_{m}$ on $\mathfrak{H}=\sum_{n=0}^{\infty} \oplus \mathcal{K}$ by $V_{n}=U_{+}$and $V_{m}=\hat{A}_{0}+U_{+}^{M} \hat{A}_{1}$ where for $\left(x_{0}, x_{1}, x_{2}, \cdots\right) \in \mathcal{H}$,

$$
U_{+}\left(x_{0}, x_{1}, x_{2}, \cdots\right)=\left(0, x_{0}, x_{1}, x_{2}, \cdots\right)
$$

and $\hat{A}_{i}\left(x_{0}, x_{1}, x_{2}, \cdots\right)=\left(A_{i} x_{0}, A_{\imath} x_{1}, A_{i} x_{2}, \cdots\right)$ for $i=0,1$. Clearly $V_{n}$ and $V_{m}$ are unilateral shifts of multiplicity $n$ and $m$ respectively.

Claim 1. $V_{n}^{m}=V_{m}^{n}$.

Claim 2. $\operatorname{dim}\left(\left(V_{n} \mathfrak{F C}\right)^{\perp} \cap\left(V_{m} \mathfrak{F C}\right)^{\perp}\right)=1$.

Let us assume these two facts for the moment, and show that $V_{n}$ and $V_{m}$ cannot commute with a unilateral shift of multiplicity one. Suppose $V_{n}$ and $V_{m}$ did commute with a unilateral shift $W$ of multiplicity one. Then

$$
W \cong M_{e}, \quad V_{n} \cong \prod_{i=1}^{n} M_{B_{\alpha_{i}}}, \quad V_{m} \cong \prod_{j=1}^{m} M_{B_{\beta_{j}}}
$$

where $B_{\alpha}$ is the Blaschke product $(\bar{\alpha} /|\alpha|)[(\alpha-z) /(1-\bar{\alpha} z)]$ for $|\alpha|<1$ (see Lemma 5 in [3]). But since $V_{n}^{m}=V_{m}^{n}$

$$
\left(\prod_{i=1}^{n} M_{B_{\alpha_{i}}}\right)^{m}=\left(\prod_{j=1}^{m} M_{B_{\beta_{j}}}\right)^{n}
$$

Hence $\prod_{i=1}^{n} B_{\alpha_{i}}^{m}=\prod_{j=1}^{m} B_{\beta_{j}}^{n}$. But this contradicts Claim 2, since either there exist some $i, k$ such that $\alpha_{i} \neq \alpha_{k}$, in which case there must exist $j, l$ such that $\beta_{j}=\alpha_{i}$ and $\beta_{l}=\alpha_{k}$ and hence 


$$
\operatorname{dim}\left(\left(V_{n} \mathfrak{H C}\right) \perp \cap\left(V_{m} \mathfrak{H C}\right)^{\perp}\right) \geqq \operatorname{dim}\left(\left(M_{B_{\alpha_{i}}} M_{B_{\alpha_{k}}} H^{2}\right)^{\perp}\right)=2,
$$

or $\alpha_{i}=\alpha$ for all $i$, in which case $\beta_{j}=\beta$ for all $j$, and hence

$$
\operatorname{dim}\left(\left(V_{n} \mathcal{H C}\right)^{\perp} \cap\left(V_{m} \mathcal{H C}\right)^{\perp}\right)=\operatorname{dim}\left(\left(M_{B_{\alpha}}^{n} H^{2}\right)^{\perp}\right)=n \geqq 2 .
$$

In either case Claim 2 is contradicted. Thus $V_{n}, V_{m}$ cannot commute with a unilateral shift of multiplicity one.

Claim 2 is obvious so we now prove Claim 1 . Since $\hat{A}_{0}$ and $\hat{A}_{1}$ commute with $U_{+}$, we note that

where

$$
V_{m}^{n}=\left(\hat{A}_{0}+U_{+}^{m} \hat{A}_{1}\right)^{n}=\sum_{k=0}^{n} U_{+}^{m \cdot k} B_{k}
$$

$$
\begin{aligned}
B_{0} & =\hat{A}_{0}^{n}=0, \\
B_{1} & =\sum_{l_{1}+l_{2}=n-1} \hat{A}_{0}^{l_{1}} \hat{A}_{1} \hat{A}_{0}^{l_{2}}, \\
B_{2} & =\sum_{l_{1}+l_{2}+l_{3}=n-2} \hat{A}_{0}^{l_{1}} \hat{A}_{1} \hat{A}_{0}^{l^{2}} \hat{A}_{1} A_{0}{ }^{l_{3}}, \\
\ldots & \\
B_{n-1} & =\sum_{l_{1}+l_{2}=n-1} \hat{A}_{1}^{l_{1}} \hat{A}_{0} \hat{A}_{1}^{l_{2}}, \\
B_{n} & =\hat{A}_{1}^{n}=0 .
\end{aligned}
$$

But then $B_{1}=I$, since, for $l=0,1, \cdots, n-1$,

$$
A_{0}^{l} A_{1} A_{0}^{n-l-1}=E_{l+1},
$$

where $E_{l}\left(y_{1}, \cdots, y_{n}\right)=\left(0,0, \cdots, y_{l}, 0, \cdots, 0\right)$. To see this, merely use matrix multiplication. In addition $B_{k}=0$ for $k=2, \cdots, n-1$, because $A_{1} A_{0}^{l} A_{1}=0$ for $l \leqq n-2$, again by matrix multiplication. Thus $V_{m}^{n}=U_{+}^{m}=V_{n}^{m}$, and Claim 1 is verified.

If $n$ and $m$ are relatively prime integers then the semigroup of operators $\left\{V(g)^{*}=V_{n}^{* k} V_{m}^{* l}\right.$ for $\left.g=k n+l m, k, l \geqq 0\right\}$ is unitarily equivalent to backward translation on $l^{2}(\mathcal{S}, \boldsymbol{C})$ for $\mathcal{S}=\{k n+l m: k, l \geqq 0\}$. In fact, this provided the motivation for the examples [2]. In this case the minimal unitary extension of the isometric semigroup $\left\{V_{n}^{k} V_{m}^{l}\right\}$ consists of bilateral shifts which do commute with a bilateral shift of multiplicity one.

\section{REFERENCES}

1. A. Brown and P. R. Halmos, Algebraic properties of Toeplitz operators, J. Reine Angew. Math. 213 (1963), 89-102. MR 28 \#3350; MR 30 p. 1205. 
2. J. A. Deddens, Coisometric extensions of semigroups of operators, Thesis, Indiana University, Bloomington, Ind., 1969.

3. P. Erdös, H. S. Shapiro and A. L. Shields, Large and small subspaces of Hilbert space, Michigan Math. J. 12 (1965), 169-178. MR 31 \#2607.

4. P. A. Fillmore, Notes on operator theory, Van Nostrand, Princeton, N. J., 1970.

5. P. R. Halmos, Shifts on Hilbert space, J. Reine Angew. Math. 208 (1961), 102112. MR 27 \#2868.

University of Michigan, Ann Arbor, Michigan 48104 